\title{
Evidências empíricas das relações entre expectativas dos agentes econômicos e desemprego no Brasil ${ }^{*}$
}

\author{
Empirical Evidence of Relationships Between Economic Agent \\ Expectations and Unemployment in Brazil
}

\begin{abstract}
Rodolfo Tomás da Fonseca Nicolay e Fabiano Roberto Santos de Lima**
\end{abstract}
\begin{abstract}
Resumo: A decisão de investimento por parte dos agentes econômicos passa pela expectativa e confiança em um ambiente de negócios mais propício. Esse otimismo pode funcionar como um mecanismo que impulsiona a criação de vagas no mercado de trabalho. Nesse contexto, este artigo analisa a existência de relações de longo e curto prazos entre o desemprego, expectativas e confiança de empresários da indústria e comércio. A análise econométrica empregada foi a de estimação pelos métodos ARDL, OLS e GMM. Os resultados revelam que expectativas mais otimistas dos empresários, no longo prazo, traduzem-se em queda dos níveis de desemprego com impactos de redução de $0,48 \%$ e $0,73 \%$ para cada 1 ponto percentual de melhora nas expectativas de indústria e comércio. Para o curto prazo, não foram encontradas evidências estatisticamente significativas de que o mesmo ocorra.
\end{abstract}

Palavras-chaves: Agentes Econômicos; Desemprego; Expectativas de Empresários

\begin{abstract}
The decision of investment by economic agents depends on the confidence and expectations of a more favorable business environment. This optimism can act as a mechanism that drives job creation. In this context, this article analyzes the existence of short and long-term relationships between unemployment and expectations and the confidence of industry and commerce entrepreneurs. The econometric analysis employed was the estimation by the ARDL, OLS, and GMM methods. The results reveal that more optimistic expectations of entrepreneurs over the long term translate into falling unemployment levels with impacts of $0.48 \%$ and $0.73 \%$ of reduction for every 1 percentage point of improvement in expectations. For the short term, no statistically significant evidence was found to occur.
\end{abstract}

Keywords: Economic Agents; Unemployment; Business Expectations

JEL: C22; E24; M51

\footnotetext{
* Submissão: 10/08/2019 | Aprovação: 09/05/2020 | DOI: 10.5380/re.v42i77.68449

** Respectivamente: (1) Universidade Candido Mendes e Universidade Católica de Petrópolis | E-mail: r-nicolay@hotmail.com | ORCID: 0000-0003-1325-5136 | (2) Universidade Estácio de Sá | E-mail: fabianosdelima@gmail.com | ORCID: 0000-0001-5821-9505
} 


\section{Introdução}

A variação da taxa de desemprego é utilizada com frequência como um indicador de desempenho do mercado de trabalho e da economia e, em última instância, é ainda um balizador de sucesso ou insucesso de políticas econômicas executadas pelos governos. O Brasil vem apresentando um fraco desempenho econômico nos últimos anos, no ano de 2015, por exemplo, o PIB real apresentou retração de -3,8\% comparado ao ano anterior e em 2016 apresentou resultado de 3,6\%. Em 2017 cresceu 1\% e manteve-se o mesmo resultado em 2018.

Frente a esse cenário, observa-se a escalada do desemprego que, segundo o Instituto Brasileiro de Geografia e Estatística (IBGE), encerrou o ano de $2018 \mathrm{com}$ uma taxa de 11,6\% de desempregados. Conclui-se, portanto, que a retomada de empregos não será algo verificável de forma imediata, fazendo-se necessário ações que possam impulsionar o crescimento do produto combinando um conjunto de reformas estruturantes com políticas econômicas que favoreçam o ambiente de negócios, conforme comentam Lima e Marques (2019).

Porém, é sabido que existem outros determinantes que indicam que a taxa de desemprego não é resultado somente das políticas econômicas praticadas. Tão pouco as alterações do equilíbrio do mercado de trabalho se dão somente por mudanças de produtividade, salários e consumo. Friedman (1968) e Phelps (1968) já haviam identificado nas expectativas uma importante variável associada ao desemprego. Particularmente neste caso, as expectativas de inflação.

De um modo geral as expectativas funcionam como importantes direcionadores de consumo na sociedade, uma vez que sua variação se dá não somente frente a mudanças de renda disponível, mas também pela confiança relacionada ao futuro que os agentes possuem. Esse ponto, sob o prisma empresarial, oferece a ideia de que a decisão de investimentos e de expansão de negócios passam pela percepção e confiança que os empresários possuem em um ambiente promissor (Antonik, 2004; Potrich, et al., 2015). Dessa forma, em concordância com Caetano e Silva (2017), o ambiente pode funcionar como um mecanismo de transmissão das expectativas e confiança dos empresários para o desemprego, influenciando, ao fim, sua variação.

Nesse sentido, pouca atenção é dada à associação das taxas de desemprego com fatores expectacionais dos agentes econômicos, estes que, de fato, são os responsáveis pela decisão de contratar mão de obra. Assim sendo, o objetivo deste 
trabalho é oferecer contribuições à literatura a partir de evidências empíricas sobre as relações de longo e curto prazos entre o desemprego e as expectativas de empresários da indústria e comércio. A análise está centrada na hipótese de que ciclos econômicos de crescimento promovem a formação de expectativas otimistas por parte dos empresários, e esse positivismo é transferido ao mercado de trabalho contribuindo para mudanças no nível de desemprego da sociedade, conforme observado por Katona (1968) e Caetano e Silva (2017).

A evolução do desemprego bem como o produto e outras variáveis macroeconômicas estão sujeitos a vários tipos de choques e, nesse caso, o uso de defasagens nas variáveis pode desempenhar um papel importante, conforme sugerem De Mendonça e Oliveira (2018). Assim sendo, a abordagem econométrica neste estudo faz uso de Modelos Autorregressivos de Defasagem Distribuída (ARDL) com cointegração desenvolvida por Pesaran, Shin e Smith (2001) para identificar relações de longo prazo. As análises para o curto prazo são evidenciadas pelos métodos dos Mínimos Quadrados Ordinários (OLS) e, de forma que a análise possa ter mais robustez, pelo Método dos Momentos Generalizados (GMM).

Além desta introdução, este trabalho está dividido em mais cinco seções: a seção dois apresenta a revisão da literatura teórica e empírica; a seção três apresenta os dados utilizados no estudo e os modelos econométricos a serem desenvolvidos; a quarta seção reporta os testes aplicados, a análise e discussão dos resultados; e a quinta seção revela as considerações finais do estudo.

\section{Revisão de literatura}

Não há concordância sobre qual teoria que melhor descreva as causas do fenômeno desemprego no mercado de trabalho brasileiro (Gouveia e Feistel, 2013; Lopes, 1982; Proni, 2013, 2014; Sachsida, 2013; Sachsida, Schettini e Gouvêa, 2017; Triches e Feijó, 2017; Zylbernstajn e Neto, 1999). Sachsida (2013) comenta que isso é devido a: i) às aproximações e proxies que diversos autores usam ao determinarem as variáveis mais apropriadas ao estudo do desemprego; ii) à problematização dos dados e; iii) devido aos diversos métodos econométricos utilizados nas estimações.

Estudos que abordam o tema são centrados em três grupos. $\mathrm{O}$ primeiro busca aprimorar o conceito de desemprego e especificar como este deve ser operacionalizado empiricamente. O segundo busca, a partir de critérios de 
segmentação demográfica, determinar a estrutura da taxa de desocupação; e, por fim, um último grupo estuda a taxa de desemprego agregada partindo da avaliação dos determinantes macroeconômicos que o compõe (Barros, Camargo e Mendonça, 1997). Em particular esse material está alinhado com o terceiro grupo.

É recorrente a utilização de variáveis como o PIB, a inflação, a taxa de juros, salários e câmbio na literatura empírica sobre desocupação. Variáveis expectacionais, normalmente, são aplicadas em estudos ligados ao consumo. No caso de investigações do mercado de trabalho, as únicas variáveis inseridas em pesquisas acerca do desemprego que traduzem as perspectivas com relação ao futuro são as expectativas de inflação.

Há farto material disponível que utilizam tais expectativas associadas ao desemprego, como, por exemplo, em Areosa, McAleer e Medeiros (2011), Mendonça, Sachsida e Medrano (2012), Sachsida, Schettini e Gouvêa (2017) e Triches e Feijó (2017).

Expectativas estão ligadas a incertezas que, por princípio, faz com que os agentes alteram seu comportamento, originando estratégias e ações que se tornam racionais e inteligíveis de acordo com seu julgo (Keynes, 1936; Potrich et al., 2015). E, em acordo com Carrol (1997), Ludvigson (2004), Dathein (2005) e Silva (2009), a sensibilidade dos agentes diante das incertezas faz com que sejam construídos estoques de reservas financeiras para se precaverem contra eventualidades e optem por não realizarem investimentos. Essa situação, portanto, torna a incerteza um determinante na composição do desemprego (Caggiano, Castelnuovo e Groshenny, 2014).

Contudo, não há como ser assertivo com relação às decisões e escolhas que os agentes econômicos tomam. As correntes de pensamento econômico abordam o papel das expectativas e desemprego de forma particular. A teoria Clássica, por exemplo, desconsidera as implicações das expectativas sobre as variáveis e as decisões econômicas. A noção de incerteza e seus efeitos junto aos agentes econômicos foi aprofundada com a corrente pós-keynesiana e os Novos Clássicos contribuiram com a formulação da Hipótese das Expectativas Racionais, na qual há uma minimização do papel das incertezas na formação das expectativas geradas no mercado. Ela afirma que os desvios do equilíbrio geral e do pleno emprego só podem ser causados por erros casuais, porém tais erros por si só não responderiam 
pelas flutuações cíclicas observadas no nível de atividade, emprego e em todas as principais variáveis econômicas (Garcia, 1999).

Além disso, é importante ressaltar que fatores políticos, mudança de rumos da economia praticada por um governo, escândalos públicos ou mesmo eleições também impactam fortemente os índices de confiança, podendo gerar "sentimentos" que influenciam a economia real via efeito contágio das expectativas dos agentes (Malgarini e Margani, 2007).

Nesse sentido, pouca atenção é dada à associação do desemprego com fatores expectacionais dos agentes econômicos, os que verdadeiramente são os responsáveis pela decisão de contratar mão de obra. Um esforço é realizado por Caleiro (2006) ao relacionar confiança do consumidor e desemprego em Portugal utilizando a metodologia Fuzzi. O trabalho revelou que desemprego e a confiança do consumidor são indissociáveis, onde as variáveis apresentaram relação negativa. Contudo não investiga causalidade ou o efeito destas sobre o empresariado.

Inserindo o produto às estimações e com a aplicação de um VAR Ortogonal, Caleiro (2007) analisa as funções de impulso-resposta e promove contribuições adicionais aos estudos anteriormente realizados indicando relações existentes entre os níveis de crescimento, o desemprego e confiança em Portugal.

Sum (2012) utilizou um VAR irrestrito para verificar empiricamente como a taxa de desemprego reage à confiança na economia dos EUA e, com dados mensais compreendidos entre 1978 a 2012, encontrou evidências de que a taxa de desemprego responde negativamente a choques na confiança nos negócios e na confiança do consumidor.

O trabalho de De Mendonça e Oliveira (2018) buscou evidenciar empiricamente a relação entre confiança das empresas e o coeficiente de Okun com dados de 15 países da OCDE no período de 2001 a 2016. Aplicando o método SysGMM, suas conclusões apontam para que em se persistindo os níveis de confiança das empresas pode haver alterações no vínculo entre desemprego e produto.

No campo das incertezas associadas à desocupação, Caggiano, Castelnuovo e Groshenny (2014) investigaram como choques de incerteza impactaram a dinâmica do desemprego nos Estados Unidos durante o período de recessão pós Segunda Guerra Mundial. Com a utilização de um modelo STVAR, concluem que modelos lineares podem subestimar o resultado dos choques sobre o desemprego 
e identificaram que os choques de incerteza têm maior impacto sobre o desemprego que choques da política monetária.

A Relação entre a incerteza e o desemprego também foi alvo da investigação de Netšunajev e Glass (2017), onde buscaram comparações entre os efeitos da relação nos EUA e na região da área do Euro. Os autores aplicaram um VAR estrutural bayesiano e concluíram que existem evidências de relações negativas entre os choques de incerteza e desemprego, onde, para os EUA, o mercado de trabalho tende a reagir aos choques da incerteza local com uma magnitude maior, porém absorve o choque mais rapidamente.

Há, portanto, certo consenso nos resultados sobre a relação de confiança ou incertezas com desemprego. Abre-se uma oportunidade para investigar no caso brasileiro se o otimismo ou pessimismo dos agentes econômicos é transferido ao mercado de trabalho com a antecipação por parte desses agentes na geração de novos postos de trabalhos.

\section{Análise empírica}

\subsection{Variáveis do estudo}

Os dados estão compreendidos entre março de 2012 a maio de 2019 com disposição mensal e utiliza-se especificação funcional logarítmica nas variáveis. Essa ação oferece a vantagem de suavização de variância das séries, reduzindo o problema de heterocedasticidade1. As variáveis adotadas na pesquisa estão descritas na Tabela 1.

\footnotetext{
${ }^{1}$ Embora não seja usual a aplicação de logaritmos em taxas, é possível encontrar sua utilização em trabalhos sobre desemprego como em Blanchflower e Oswald (1994), Rendtel e Schwarze (1996), Hilbert (2008) e Netšunajev e Glass (2017). Para outras variáveis que se apresentam como sendo percentuais, Usman e Elsalih (2018) aplicam logaritmos também na taxa de câmbio associado ao desemprego. Sarel (1996), Ghosh e Phillips (1998), Khan e Ssnhadji (2001) utilizaram log na taxa de inflação para melhor ajustamento aos modelos utilizados (Wooldridge, 2007).
} 
Tabela 1 - Variáveis do estudo

\begin{tabular}{|c|c|c|c|}
\hline Variável & Série & Fonte & Periodicidade \\
\hline $\ln D E S$ & Taxa de desocupação - PNADC & Série 24369 - BCB & Mensal \\
\hline $\ln P R O D$ & $\begin{array}{l}\text { Índice de atividade econômica - } \\
\text { IBC-Br }\end{array}$ & $\mathrm{BCB}$ & Mensal \\
\hline $\operatorname{lnINFL}$ & $\begin{array}{l}\text { Expectativa média de inflação - } \\
\text { IPCA ( } 12 \text { meses) }\end{array}$ & BCB - Ipeadata & Mensal \\
\hline $\ln T A X$ & Selic & Série 4189 - BCB & Mensal \\
\hline $\operatorname{lnIND}$ & $\begin{array}{l}\text { Confiança do empresário industrial - } \\
\text { ICEI: Expectativas }\end{array}$ & CNI - Ipeadata & Mensal \\
\hline $\ln C O M$ & $\begin{array}{l}\text { Confiança do Empresário do } \\
\text { Comércio - ICEC }\end{array}$ & $\mathrm{CNC}$ & Mensal \\
\hline
\end{tabular}

Fonte: Elaboração própria.

a) Desemprego $(\boldsymbol{l n} \boldsymbol{D E} \boldsymbol{S})$ - A taxa de desemprego utilizada é a fornecida pelo IBGE através da Pesquina Nacional por Amostra de Domicílios, disponível no sítio do Banco Central do Brasil.

b) IBC-Br ( InPROD) - Adota-se o IBC-Br com ajuste sazonal como proxy para o PIB. O índice incorpora a trajetória de variáveis consideradas como proxies para o desempenho de todos os setores da economia.

c) Inflação (InINFL) - Utilizou-se como proxy da inflação nacional a expectativa de inflação capturada no último dia útil de cada mês para os doze meses subsequentes, considerando que, após a divulgação da inflação oficial verificada em um período, os agentes têm a possibilidade de reunir todas as informações necessárias para formular sua expectativa de inflação para os períodos futuros.

d) Taxa de juros $(\boldsymbol{l n} \boldsymbol{T A X})$ - A taxa de juros Selic acumulada no mês, anualizada, serve como parâmetro da taxa de juros no país.

e) Expectativas do empresário da indústria (InIND) - Expressa as expectativas dos empresários industriais acerca do desenvolvimento de seus negócios frente ao desempenho da economia. É medido pela Confederação Nacional da Indústria com base em questões referentes a condições atuais comparadas com os últimos seis meses. A metodologia utilizada é a de pontuação que varia entre 0 e 100 e quanto maior o índice, melhores são as expectativas dos empresários em relação à economia e aos seus negócios.

f) Confiança do empresário do comércio ( $\operatorname{lnCOM}$ - O índice de confiança do empresário do comércio (ICEC) é indicador mensal antecedente, apurado pela Confederação Nacional do Comércio entre os 
tomadores de decisão das empresas do varejo para detectar as tendências das ações do setor do ponto de vista do empresário.

O esquema empírico da análise é demonstrado na Figura 1 abaixo:

Figura 1 - Esquema de Análise do Estudo

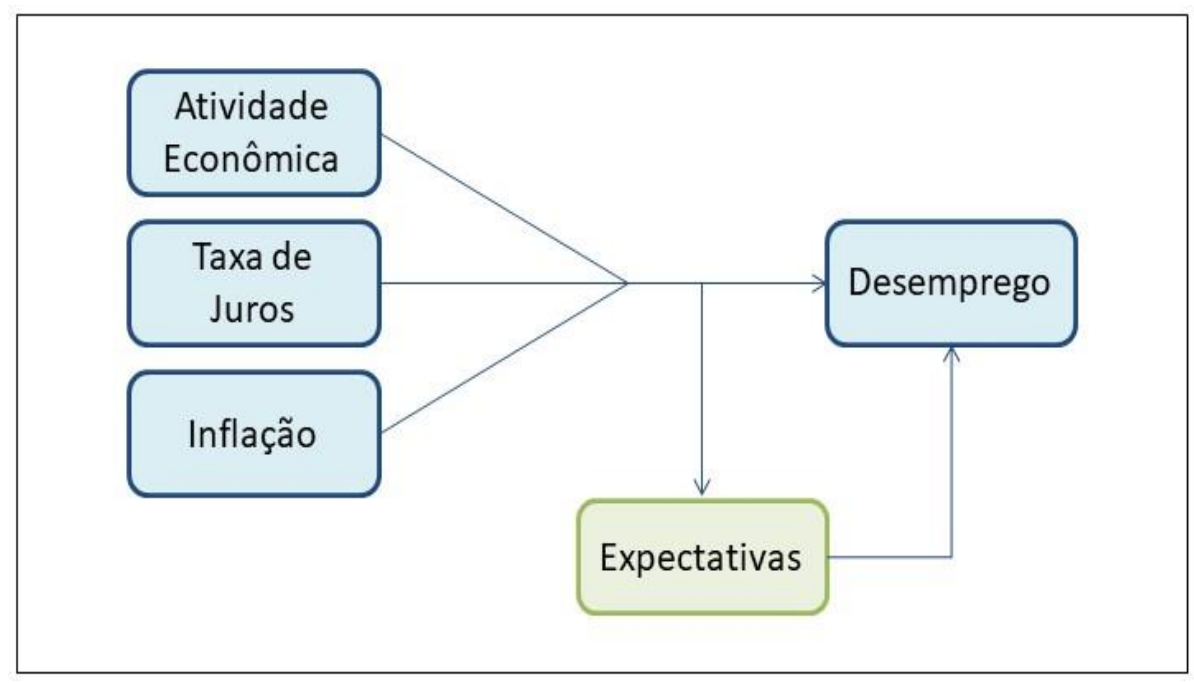

Fonte: Elaboração própria.

A Figura 2 aponta para comportamentos similares ao longo do tempo entre confiança do empresário, do comércio e expectativas da indústria em que, dados eventos importantes na política e economia no período, moveram as variáveis expectacionais para condições em que, ora deterioravam-se, como por exemplo a possibilidade de vitória na eleição presidencial de um determinado candidato, e ora melhoravam, quando da possibilidade de saída e efetivo impeachment da presidente da República ao final de agosto de 2016. 


\section{Figura 2 - Evolução das Expectativas dos Agentes Econômicos}

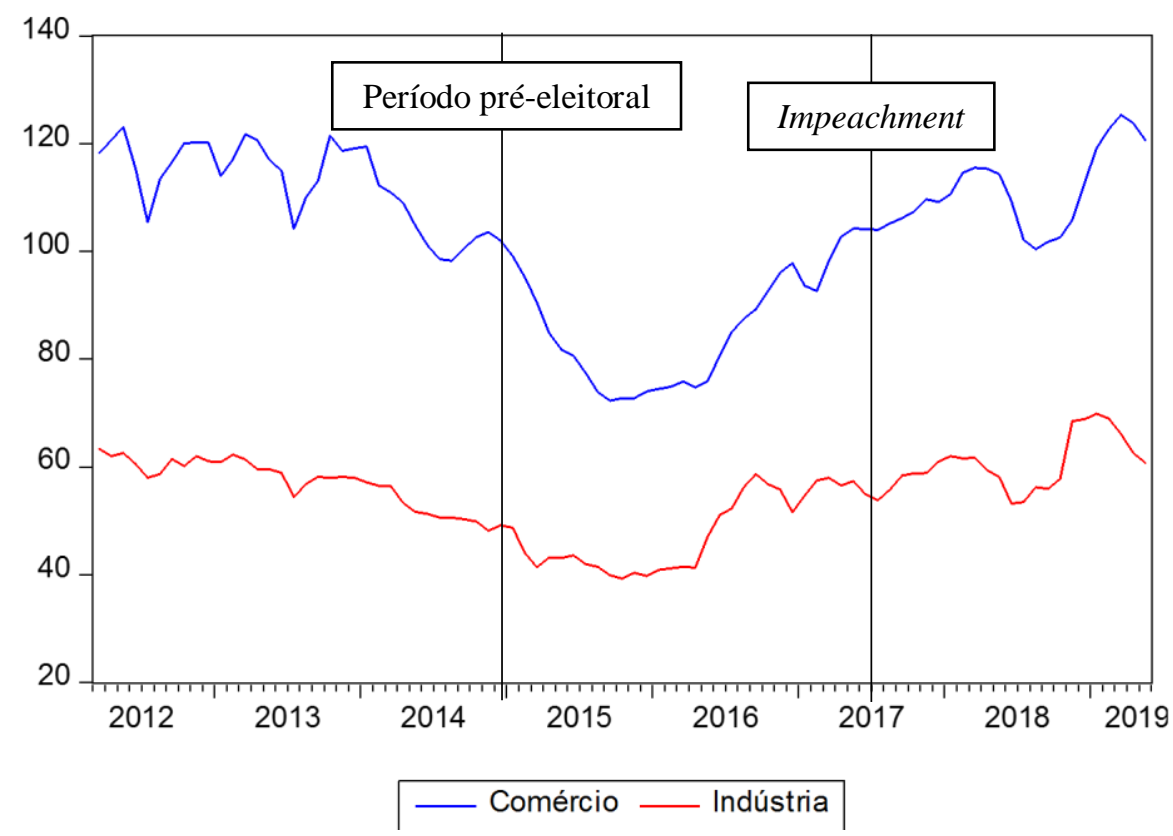

Fonte: CNC e Ipeadata.

Numa comparação estática (Figura 3), é possível observar que, do ano de 2014 a 2015, os níveis de desemprego seguem em ascensão enquanto as expectativas pioram. Porém, a partir de 2016, nitidamente com reversão dos indicadores expectacionais, ao contrário, a taxa de desemprego também continuou subindo, revelando dois pontos: primeiro, a demora desse indicador do mercado de trabalho em responder ao ciclo e, em segundo lugar, a uma possível redução de poder de transferência das expectativas dos agentes ao mercado de trabalho na geração de empregos no curto prazo.

Com base nessa observação, confirma-se para o período um componente expectacional derivado de eventos políticos e econômicos contemporâneos, conforme afirmam Malgarini e Margani (2007), e que os efeitos positivos da melhora das expectativas em relação ao futuro podem não estar mais sendo "capturados" pelo mercado de trabalho, restando investigar acerca de seus impactos de curto e longo prazo. 
Figura 3 - Comparação entre as expectativas dos agentes e desemprego

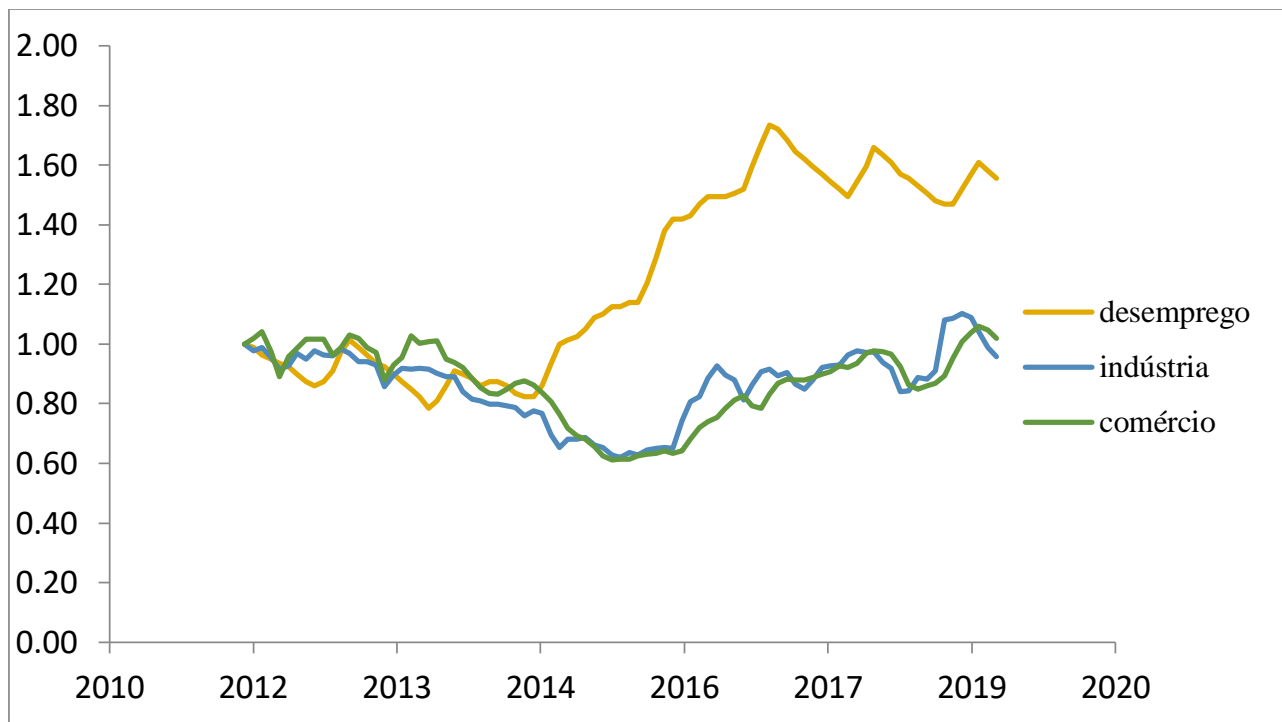

Fonte: CNC, Ipeadata e BCB (IBGE-PNADC).

Nota: Dados convertidos em números índices.

\subsection{Modelo empírico}

\subsubsection{Cointegração para modelos ARDL}

O Teste de Limites de Pesaran, Shin e Smith (2001) é um teste de cointegração que se baseia em modelos ARDL. Seu objetivo é verificar a existência de relação de longo prazo entre as variáveis, não sendo necessário analisar a ordem de integração destas, desde que as variáveis possuam ordem de integração menor do que dois. Portanto, podem todas as séries serem integradas de ordem $0[\mathrm{I}(0)]$, de ordem $1[\mathrm{I}(1)]$ ou mutuamente cointegradas.

O teste de cointegração é realizado com base na dinâmica do modelo de correção de erros (ECM), e, de acordo com Pesaran, Shin e Smith (2001), partindose de um vetor autorregressivo de ordem p[VAR(p)], pode-se especificar o ECM, conforme descreve a Equação 1.

$$
\Delta Y_{t}=\beta_{0}+\sum_{i=1}^{n} \beta_{j} \Delta y_{t-j}+\sum_{t=0}^{p} \delta_{j} \Delta x_{t-j}++\varphi y_{t-1}+\varphi x_{t-1}+\varepsilon_{t}
$$

em que $\Delta Y$ t representa a primeira diferença; $\beta 0$ é o termo da constante; $\beta \mathrm{j}$ e, $\delta \mathrm{j}$ são os parâmetros de longo prazo; $\varphi$ representa parâmetros de curto prazo e $\varepsilon t$ um processo de ruído branco, $\mathrm{N}(0, \boldsymbol{\sigma} 2)$, identicamente, independentemente distribuída. 
A análise da relação entre as variáveis deste estudo se dá por meio da estimação de três modelos ARDL. O modelo 1 sugere a hipótese de que o volume de atividade econômica nacional, a inflação e taxa de juros possuem influência na determinação do nível de desemprego brasileiro. Ao modelo 2 é acrescida a confiança que o empresário da indústria tem no desempenho da economia no país, e no modelo 3 a confiança do empresário industrial é substituída pela confiança do empresário do segmento do comércio, conforme descrição abaixo:

\section{Modelo 1:}

$$
\begin{aligned}
& \Delta \ln D E S_{t}=\beta_{0}+\sum_{j=1}^{n} \beta_{j} \Delta \ln D E S_{t-j}+\sum_{t=0}^{n} \omega \Delta \ln P R O D_{t-j}+\sum_{t=0}^{m} \partial \Delta \ln I N F L_{t-j}+ \\
& \sum_{t=0}^{n} \delta \Delta \ln T A X_{t-j}+\beta_{1} \ln D E S_{t-1}+\beta_{2} \ln P R O D_{t-1}+\beta_{3} \ln I N F L_{t-1}+\beta_{4} \ln T A X_{t-1}+ \\
& \varepsilon_{t}
\end{aligned}
$$

\section{Modelo 2:}

$$
\begin{aligned}
& \Delta \ln D E S_{t}=\beta_{0}+\sum_{j=1}^{n} \beta_{j} \Delta \ln D E S_{t-j}+\sum_{t=0}^{n} \omega \Delta \ln P R O D_{t-j}+\sum_{t=0}^{m} \partial \Delta \operatorname{lnINFL_{t-j}+} \\
& \sum_{t=0}^{n} \delta \Delta \ln T A X_{t-j}+\sum_{t=0}^{n} \psi \Delta \ln I N D_{t-j}+\beta_{5} \ln D E S_{t-1}+\beta_{6} \ln P R O D_{t-1}+ \\
& \beta_{7} \ln I N F L_{t-1}+\beta_{8} \ln T A X_{t-1}+\beta_{9} \ln I N D_{t-1}+\varepsilon_{t}
\end{aligned}
$$

\section{Modelo 3:}

$$
\begin{aligned}
& \Delta \operatorname{lnDES} S_{t}=\beta_{0}+\sum_{j=1}^{n} \beta_{j} \Delta \ln D E S_{t-j}+\sum_{t=0}^{n} \omega \Delta \ln P R O D_{t-j}+\sum_{t=0}^{m} \partial \Delta \ln I N F L_{t-j}+ \\
& \sum_{t=0}^{n} \delta \Delta \ln T A X_{t-j}+\sum_{t=0}^{n} \tau \Delta \ln C O M_{t-j}+\beta_{10} \ln D E S_{t-1}+\beta_{11} \ln P R O D_{t-1}+ \\
& \beta_{12} \operatorname{lnINFL_{t-1}}+\beta_{13} \ln T A X_{t-1}+\beta_{14} \operatorname{lnCOM}_{t-1}+\varepsilon_{t}
\end{aligned}
$$

\subsubsection{Métodos OLS e GMM}

A análise empírica para as relações de curto prazo entre as variáveis se dá com base nas estimações dos métodos OLS e GMM. Este último com o objetivo de oferecer maior robustez aos resultados. Diferentemente da abordagem ARDL, nesses dois métodos, é importante atender a condição de que as séries sejam integradas de ordem 0 , pois do contrário necessitam ser diferenciadas sob o cuidado de não gerar regressões espúrias. Além desse cuidado, ao se trabalhar com séries temporais deve ser atentado aos problemas relacionados à endogeneidade e simultaneidade nos regressores. Particularmente para o GMM, a solução ao problema se dá pela utilização de instrumentos defasados em um ou mais períodos, conforme Johnston (1984) sugere. 
Os modelos dinâmicos a serem utilizados são os mesmos apresentados anteriormente, no total de 3 equações. As variáveis instrumentais escolhidas foram datadas do período t-1 ou anterior, conforme apresentado na Tabela 7 (ver Apêndice). É utilizado ainda o teste $\mathrm{J}$ para verificar se os instrumentos respeitam a hipótese de ortogonalidade, conforme sugere Hansen (1982), e, por último, com objetivo de extrair o componente de ciclos de negócios das séries de tempo utilizadas, foi aplicado o filtro HP (Hodrick e Prescott, 1997) em todas as séries para todas estimações realizadas.

\section{Análise e discussão dos resultados}

\subsection{Teste de estacionariedade}

A condição primária para o teste de cointegração ARDL é a garantia de que as séries utilizadas sejam integradas $\mathrm{I}(0), \mathrm{I}(1)$ ou mutuamente cointegradas, sendo impossível a utilização dessa abordagem caso as séries sejam I(2).

Com a aplicação dos testes Augmented Dickey-Fuller (ADF) e PhillipsPerron (PP), ambos com a hipótese nula de que a série não seja estacionária e adotando-se o critério de aceitação de raiz unitária a $10 \%$ de significância, não foram encontradas evidências de variáveis I(2), conforme apresentado na Tabela 7 (Ver Apêndice).

\subsection{Estimações dos modelos ARDL}

As estimações dos modelos seguem a construção $\operatorname{ARDL}(a, b, c, d, e)$, de onde entende-se " $a$ " como a quantidade de defasagens da variável dependente, enquanto que as demais $-b, c, d, e$ - referem-se ao número de defasagens das demais variáveis utilizadas sequenciadas.

Utilizou-se o critério de Akaike para as determinações de defasagens automáticas com matriz Newey-West para corrigir possíveis efeitos de correlação dos termos de erro nas regressões construídas. O número máximo de lags automáticos para cada parâmetro foi 12 e a ordem das variáveis segue o anteriormente descrito e apresentado para cada equação.

A estabilidade dos parâmetros das regressões seguiu os testes de Soma Cumulativa dos Resíduos Recursivos (CUSUM) e Soma Cumulativa dos Quadrados dos Resíduos Recursivos (CUSUMSQ) proposto por Brown, Durbin e 
Evans (1975). Ambos os testes com finalidade de monitorar a variabilidade do processo, uma vez que a hipótese nula deste é a de estabilidade dos coeficientes calculados a 5\% de significância. Em todos os casos evidenciou-se a estabilidade dos parâmetros no período analisado2, conforme Figura 4 (Apêndice).

Os testes de cointegração para todas estimações revelaram uma possível relação de curto ou de longo prazo entre as variáveis. Todos modelos apresentaram F-estatísticos acima das fronteiras superiores I[1], rejeitando, portanto, a hipótese nula a $1 \%$ de que não há cointegração entre as variáveis nos modelos apresentados, conforme revela a Tabela 2 .

Tabela 2 - Teste de Cointegração ARDL - Teste dos Limites (Bounds)

\begin{tabular}{lccc}
\hline \multirow{2}{*}{ Parâmetros } & Modelo 1 & Modelo 2 & Modelo 3 \\
& ARDL $(12,11,12,10)$ & $(12,10,12,11,11)$ & $(12,09,12,10,12)$ \\
\cline { 2 - 4 } & Significância 1\% & Significância 1\% & Significância 1\% \\
\hline F-estatístico & 8.06 & 13.72 & 18.32 \\
Limite Inferior I[0] & 5.17 & 4.40 & 4.40 \\
Limite Superior I[1] & 6.36 & 5.72 & 5.72 \\
Critério de seleção & $\mathrm{R}^{2}$ Ajustado & $\mathrm{R}^{\mathbf{2}}$ Ajustado & $\mathrm{R}^{2}$ Ajustado \\
Lags máximos & 12 & 12 & 12 \\
\hline
\end{tabular}

Fonte: Elaboração própria a partir dos dados da pesquisa.

\subsubsection{Análise dos Coeficientes da Estimação ARDL}

A significância dos coeficientes para o longo prazo estão reportados na Tabela 4. Todos os modelos apresentaram resíduos adequados, com ausência de autocorrelação serial e heterocedasticidade. Em todos os 3 casos evidencia-se o impacto que o desemprego sofre pelo nível de atividade no país, pela inflação e pela taxa de juros Selic, esta última impacta positivamente a taxa de desemprego. Como em Dezordi, (2011), Dos Santos (2016), Gouveia e Feistel, (2015), Palma e Ferreira (2017), Medeiros, Portugal e Aragón (2017), os resultados obtidos são consistentes com a literatura existente e validam a lei de Okun e a curva de Phillips para o caso brasileiro no período estudado.

\footnotetext{
${ }^{2}$ Foram testados junto aos modelos os eventos políticos e econômicos através de uma variável dummy que capturou efeitos da crise política brasileira iniciada no ano de 2014 até o impeachment da presidente Dilma Rousseff. Como os resultados não foram estatisticamente significativos com sua inserção nem para o curto ou longo prazos, optou-se por não reportar tais estimativas.
} 
Tabela 3 - Estimação dos Modelos ARDL

\begin{tabular}{|c|c|c|c|}
\hline \multicolumn{4}{|c|}{ Variável dependente: Desemprego $(\ln D E S)$} \\
\hline Séries & $\begin{array}{c}\text { Modelo 1 } \\
(12,11,12, \\
10)\end{array}$ & $\begin{array}{c}\text { Modelo 2 } \\
(12,10,12,11, \\
11)\end{array}$ & $\begin{array}{c}\text { Modelo 3 } \\
(12,09,12,10, \\
12)\end{array}$ \\
\hline \multirow[t]{3}{*}{$\ln P R O D$} & $-6.5234 * * *$ & $-3.3237 * * *$ & $-1.1204 * *$ \\
\hline & $(0.3536)$ & $(0.3329)$ & $(0.5152)$ \\
\hline & {$[-18.4467]$} & {$[-9.9830]$} & {$[-2.1744]$} \\
\hline \multirow[t]{3}{*}{$\operatorname{lnINFL}$} & $-1.1019 * * *$ & $-1.1665 * * *$ & $-1.0210 * * *$ \\
\hline & $(0.1710)$ & $(0.0431)$ & $(0.0460)$ \\
\hline & {$[-6.4428]$} & {$[-27.0544]$} & {$[-22.1628]$} \\
\hline \multirow[t]{3}{*}{$\ln T A X$} & $0.6237 * * *$ & $0.3303 * * *$ & $0.1594 * * *$ \\
\hline & $(0.1276)$ & $(0.0269)$ & $(0.0270)$ \\
\hline & [4.4849] & [12.2768] & [5.8856] \\
\hline \multirow{3}{*}{$\ln I N D$} & - & $-0.4869 * * *$ & - \\
\hline & - & $(0.0630)$ & - \\
\hline & - & {$[-7.7208]$} & - \\
\hline \multirow[t]{3}{*}{$\ln C O M$} & - & - & $-0.7313 * * *$ \\
\hline & - & - & $(0.0883)$ \\
\hline & - & - & {$[-8.2733]$} \\
\hline \multicolumn{4}{|c|}{ Significâncias Totais dos Modelos } \\
\hline $\mathrm{R}^{2}$ & 0.9966 & 0.9990 & 0.9989 \\
\hline $\mathrm{R}^{2}$ Ajustado & 0.9900 & 0.9947 & 0.9945 \\
\hline F-estatístico & 298.573 & 232.282 & 225.177 \\
\hline Prob (F-estatístico) & 0.0000 & 0.0000 & 0.0000 \\
\hline \multicolumn{4}{|c|}{ Diagnóstico dos Resíduos } \\
\hline \multirow{3}{*}{$\begin{array}{c}\text { Jarque-Bera } \\
\text { Autocorrelação (LM-test) } \\
\text { Heterocedasticidade } \\
\text { (ARCH) }\end{array}$} & 0.2566 & 0.6427 & 0.5427 \\
\hline & 0.4011 & 0.3317 & 0.5157 \\
\hline & 0.9878 & 0.7952 & 0.2096 \\
\hline
\end{tabular}

Fonte: Elaboração própria.

Notas: Coeficientes em negrito. * Significativo a $10 \%$; *** Significativo a $1 \%$. Entre parênteses ( ): Desvio Padrão. Entre colchetes [ ]: Estatística-t.

Os modelos acrescidos da expectativa da indústria e confiança do comércio apresentaram evidências da existência de relação negativa com o desemprego. No primeiro caso, alterações em 1 ponto percentual nas expectativas do empresariado da indústria impactam negativamente na variação da taxa real de desemprego em 0,48\%. Já quando analisado o Modelo 3, percebe-se que a relação da confiança do empresário do comércio com a variável dependente também é negativa e indica 
uma variação de $0,73 \%$ na taxa real de desemprego a cada mudança em 1 ponto percentual no índice de confiança.

$\mathrm{O}$ valor de $\mathrm{R}^{2}$ também revela ajuste satisfatório em que pode se afirmar que as oscilações das variáveis analisadas conjuntamente em cada modelo explicam em $99 \%$ o resultado e as alterações da taxa de desemprego.

\subsubsection{Dinâmica de Curto Prazo e Correção de Erros (ECM-ARDL)}

As relações de longo prazo não eliminam a possibilidade de alterações de resultados dado a existência de choques de curto prazo. Com isso, para que a relação de cointegração se mantenha, é necessário um mecanismo de correção desses choques para que se retorne à dinâmica de longo prazo. A Tabela 4 apresenta os ajustes de curto prazo que reportam as estimações dos vetores de correção de erros dos modelos ARDL.

Tabela 4 - Mecanismo de Correção de Erros

\begin{tabular}{cccc}
\hline & Modelo 1 & Modelo 2 & Modelo 3 \\
ARDL & $(12,11,12,10)$ & $(12,10,12,11,11)$ & $(12,09,12,10,12)$ \\
\hline CointEq(-1) & $-0.4936^{* * *}$ & $-1.4175^{* * *}$ & $-1.3388^{* * *}$ \\
\hline
\end{tabular}

Fonte: Elaboração própria.

Nota: *** Significativo a $1 \%$.

O ECM, para todos os casos, apresentou resultado negativo e estatisticamente significativo. Essa condição revela que haverá convergência no modelo com significativa relação para o longo prazo, indicando ainda que a velocidade de ajustamento para o equilíbrio anterior ao choque se dá de forma mais acelerada quando levado em consideração as expectativas dos empresários da indústria.

\subsection{Estimação dos modelos por OLS e GMM}

Nas estimações via OLS apresentadas na Tabela 5, todos os coeficientes dos modelos propostos seguiram a mesma direção em sinais e magnitude esperados. As variáveis que apresentaram indícios de ocasionarem impacto negativo no desemprego foram o produto e a inflação. A taxa de juros Selic se relaciona positivamente com o desemprego. Esses resultados estão em acordo com a literatura econômica, tal qual os resultados obtidos com a estimação via ARDL. 
Tabela 5 - Estimações dos Modelos por OLS e GMM

\begin{tabular}{|c|c|c|c|c|c|c|}
\hline \multirow[b]{2}{*}{ Variável } & \multicolumn{3}{|c|}{ OLS } & \multicolumn{3}{|c|}{ GMM } \\
\hline & Modelo 1 & Modelo 2 & Modelo 3 & Modelo 1 & Modelo 2 & Modelo 3 \\
\hline \multirow[t]{3}{*}{$\ln P R O D$} & $0.4739 * *$ & $-0.4869 *$ & $-0.4670 *$ & $-0.7489 * *$ & $-0.7168 *$ & $1.2651^{*} * * *$ \\
\hline & $(0.2351)$ & $(0.2556)$ & $(0.2281)$ & $(0.3514)$ & $(0.4021)$ & $(0.2784)$ \\
\hline & {$[-2.0152]$} & {$[-1.9051]$} & {$[-2.0468]$} & {$[-2.1311]$} & {$[-1.7826]$} & {$[-4.5430]$} \\
\hline \multirow[t]{3}{*}{$\operatorname{lnINFL}$} & $\overline{-}^{-}$ & $\overline{-}$ & $\overline{-}$ & $\begin{array}{c}- \\
0.1 \% * * *\end{array}$ & $\begin{array}{c}- \\
0.96 * * *\end{array}$ & $\begin{array}{c}- \\
0=1\end{array}$ \\
\hline & $\begin{array}{c}\mathbf{0 . 3 2 6 3} * * \\
(0.1389)\end{array}$ & $\begin{array}{c}\mathbf{0 . 3 4 9 9} * * * \\
(0.1657)\end{array}$ & $\begin{array}{c}\mathbf{0 . 3 8 2 6} * * \\
(0.1582)\end{array}$ & $\begin{array}{c}\mathbf{0 . 2 8 2 1} * * * * \\
(0.0990)\end{array}$ & $\begin{array}{c}\mathbf{0 . 3 6 4 2} * * * * \\
(0.1236)\end{array}$ & $\begin{array}{c}\mathbf{0 . 5 4 0 1} \cdots * * * \\
(0.1682)\end{array}$ \\
\hline & {$[-2.3481]$} & {$[-2.0811]$} & {$[-2.4175]$} & {$[-2.8493]$} & {$[-2.9469]$} & {$[-3.2100]$} \\
\hline \multirow[t]{3}{*}{$\ln T A X$} & $0.2137 * *$ & $0.2130 * *$ & $0.1825^{*}$ & $0.1895 *$ & $0.2251 * * *$ & $0.1744 *$ \\
\hline & $(0.1022)$ & $(0.1034)$ & $(0.1053)$ & $(0.1126)$ & $(0.0685)$ & $(0.0903)$ \\
\hline & [2.0895] & [2.0584] & [1.7332] & [1.6829] & [3.2824] & [1.9313] \\
\hline \multirow[t]{3}{*}{$\operatorname{lnIND}$} & - & -0.0305 & - & - & -0.0249 & - \\
\hline & - & $(0.1508)$ & - & - & $(0.1265)$ & - \\
\hline & - & {$[-0.2025]$} & - & - & {$[-0.1971]$} & - \\
\hline \multirow[t]{3}{*}{$\ln C O M$} & - & - & -0.1497 & - & - & -0.1733 \\
\hline & - & - & $(0.1756)$ & - & - & $(0.1652)$ \\
\hline & - & - & {$[-0.8525]$} & - & - & {$[-1.0491]$} \\
\hline \multicolumn{7}{|c|}{ Significâncias Totais dos Modelos } \\
\hline $\mathrm{R}^{2}$ & 0.1655 & 0.1951 & 0.2149 & 0.1507 & 0.1460 & 0.0821 \\
\hline $\mathrm{R}^{2}$ Ajustado & 0.1354 & 0.1559 & 0.1767 & 0.1176 & 0.1011 & 0.0337 \\
\hline F-estatístico & 6.6795 & 4.9715 & 5.6144 & - & - & - \\
\hline $\begin{array}{c}\text { Prob (F- } \\
\text { estatístico) }\end{array}$ & 0.0004 & 0.0012 & 0.0004 & - & - & - \\
\hline J-estatístico & - & - & - & 13.375 & 14.782 & 12.202 \\
\hline Prob (J-estatístico) & - & - & - & 0.2034 & 0.4671 & 0.4295 \\
\hline
\end{tabular}

Fonte: Elaboração própria

Notas: Coeficientes em negrito. * Significativo a 10\%; ** Significativo a 5\%; *** Significativo a 1\%. Entre parênteses ( ): Desvio Padrão. Entre colchetes [ ]: Estatística t.

Os casos analisados possuem resultados similares entre si com relação negativa entre produto e desemprego oscilando de $0,46 \%$ a $0,48 \%$, dependendo do modelo analisado. Já para a variável de inflação, as evidências indicam relação também negativa com o desemprego, apresentando resultados que oscilam entre $0,32 \%$ e $0,38 \%$, dependendo do modelo analisado. A taxa de juros Selic possui relação positiva com o desemprego e pode variar, de acordo com o modelo observado, de $0,18 \%$ a $0,21 \%$. Embora o sinal obtido com os modelos que incorporam as expectativas e a confiança sejam adequados, não foi possível verificar relações estatisticamente significativas entre esses indicadores e a taxa de desemprego. 
Pela abordagem GMM (Tabela 5), os resultados obtidos acompanham os verificados no método OLS em grau e magnitude desenvolvidos no estudo. $\mathrm{Na}$ dinâmica de curto prazo, em ambas as abordagens, OLS e GMM, não foram identificadas evidências estatisticamente significativas de que a confiança dos empresários da indústria e comércio contribuam para diminuição dos níveis de desemprego no país.

\section{Considerações finais}

Este trabalho apresentou evidências empíricas das relações entre o desemprego brasileiro, variáveis macroeconômicas, expectativas e confiança dos agentes econômicos da indústria e comércio. Os dados do estudo compreendem o recorte temporal de março de 2012 a junho de 2019 e possuem periodicidade mensal. Foram utilizadas as abordagens econométricas ARDL para testar a existência de relações de longo prazo, OLS e GMM para verificar relações de curto prazo. Os resultados das estimações são consistentes com a teoria econômica, os quais puderam atestar a Lei de Okun e Curva de Phillips para o Brasil no período analisado.

As evidências apontam para existência de relação de longo prazo entre desemprego, o nível de atividade, inflação, instrumento de política monetária e as expectativas dos empresários da indústria e comércio, onde a percepção mais positiva relacionada ao futuro contribui para a redução das taxas de desemprego.

No caso de haver choques que impactem os níveis de empregos na sociedade, levando-se em consideração as expectativas do setor industrial, é possível que esse segmento se recupere com maior velocidade e retorne à condição de desemprego de equilíbrio pré-choque mais rapidamente. Essa afirmação não implica que as taxas de desemprego reduzirão imediatamente com melhoras nas expectativas e sim que as expectativas da indústria contribuam mais que a confiança do comércio para a recuperação.

Similarmente, a estimação OLS utilizada para verificar as relações de curto prazo entre desemprego e as demais variáveis deste estudo estão em concordância com a literatura econômica, contudo não foram encontradas evidências de relações estatisticamente significativas entre as expectativas, confiança do empresariado e a taxa de desemprego. Assim sendo, não parece que otimismo ou pessimismo ofereçam impacto no desemprego nacional no curto prazo. Esse resultado foi confirmado com a estimação pelo método GMM, que oferece robustez ao teste. 
Os resultados contribuem com a literatura e tornam-se relevantes pois sabendo como o desemprego responde às expectativas dos agentes, os policymakers podem direcionar ações para a tentativa de construção de surtos de otimismo de forma que este seja transferido ao mercado de trabalho.

\section{Agradecimentos}

Os autores agradecem a Ivan Salomão (Editor) e a um revisor não identificado por seus comentários muito úteis eximindo-o de qualquer responsabilidade por erros remanescentes.

\section{Referências}

ANTONIK, L. R. Análise de projetos de investimento sob condições de risco. Revista da FAE. v. 7, n. 1, 2004.

AREOSA, W.; McALEER, M.; MEDEIROS, M. Moment-based estimation of smooth transition regression models with endogenous variables. Journal of Econometrics, v. 165, n. 1, p. 100-111, 2011.

BANCO CENTRAL DO BRASIL - BCB. Índice de Atividade Econômica do Banco Central - IBC-Br. Diponível em: <https://dadosabertos.bcb.gov.br /dataset/24363-indice-de-atividade-economica-do-banco-central---ibc-br> Acesso em: 12 ago. 2019.

BANCO CENTRAL DO BRASIL - BCB. Taxa de desocupação - PNAD. Série 24369. Diponível em:<https://www3.bcb.gov.br/sgspub/consultarvalores/tela CvsSelecionarSeries.paint> Acesso em: 12 ago. 2019.

BANCO CENTRAL DO BRASIL - BCB. Taxa de juros Selic. Série 4189. Diponível em: <https://www3.bcb.gov.br/sgspub/consultarvalores/telaCvsSeleci onarSeries.paint> Acesso em: 12 ago. 2019.

BARROS, R.; CAMARGO, J.; MENDONÇA, R. Estrutura do Desemprego no Brasil. Texto para discussão n. 478. Brasilia: IPEA, 1997.

BLANCHFLOWER, D. G.; OSWALD, A. J. Estimating a Wage Curve for Britain 1973-90. The Economic Journal, v. 104, n. 426, p. 1025-1043, 1994.

BROWN, R.; DURBIN, J.; EVANS, J. Techniques for testing the constancy of regression relationships over time. Journal of the Royal Statistical Society. Series B (Methodological), v. 37, n. 2, p. 149-163, 1975. 
CAETANO, R.; SILVA, C. Determinantes da Confiança do Consumidor e Dinâmica da Política Monetária no Brasil. In: X Encontro Internacional da Associação Keynesiana Brasileira, 2017.

CAGGIANO, G.; CASTELNUOVO, E.; GROSHENNY, N. Uncertainty shocks and unemployment dynamics in U.S. recessions. Journal of Monetary Economics, v. 67, p. $78-92,2014$.

CALEIRO, A. Crescimento económico e desemprego em Portugal, uma explicação adicional para a sua relação. Documento de Trabalho n. 2007/05, Universidade de Évora, Departamento de Economia.

CALEIRO, A. How is confidence related to unemployment in Portugal? Applied Economics Letters, v. 13, n. 13, p. 887-890, 2006.

CARROLL, C. Buffer-stock saving and the life cycle/permanent income hypothesis. The Quarterly Journal of Economics, v. 112, n. 1, p. 1-55, 1997.

CONFEDERAÇÃO NACIONAL DO COMÉRCIO - CNC. Índice de Confiança do Empresário do Comércio (ICEC). Disponível em <http://cnc.org.br /editorias/economia/pesquisas/indice-de-confianca-do-empresario-do-comercioicec-maio-de-2019> Acesso em: 24 dez. 2019.

DATHEIN, R. Teorias econômicas e políticas contra o desemprego: uma avaliação das diferentes propostas. Pesquisa \& Debate, v. 16, n. 1, 2005.

DE MENDONÇA, H. F.; OLIVEIRA, D. S. P. Firms' confidence and Okun's law in OECD countries. Economic Modelling, v. 78, p. 98-107, 2019.

DEZORDI, L. L. A Lei de Okun para a economia brasileira: 2002-2010. Vitrine da Conjuntura, v. 4, n. 5, p. 1-3, 2011.

DOS SANTOS, F. S. Okun's law and labor productivity in Brazil. In: $43^{\circ}$ Encontro Nacional de Economia, ANPEC. Florianopolis, 2016.

FRIEDMAN, M. The role of monetary policy. The American Economic Review, v. $58, \mathrm{n} 1.1968$.

GARCIA, R. L. O papel da incerteza na formação das expectativas e na determinação da taxa de juros. Economia e Desenvolvimento, n. 10, 1999.

GHOSH, A.; PHILLIPS, S. Warning: inflation may be harmful to your growth. International Monetary Fund, Staff Papers, v. 45, n. 4, p. 672-710, 1998.

GOUVEIA, J. M. A.; FEISTEL, P. R. Uma aplicação da lei de Okun no Brasil (1996-2013). Revista Economia do Centro-Oeste, v. 1, n. 1, p. 81-90, 2015. 
HANSEN, L. Large sample properties of generalized method of moments estimators. Econometrica: Journal of the Econometric Society, v. 50, n. 4, p. 10291054, 1982.

HILBERT, C. Unemployment, wages, and the impact of active labour market policies in a regional perspective. Berlim: Logos Verlag Berlin GmbH, 2008.

HODRICK, R.; PRESCOTT, E. Business cycles: an empirical investigation. Journal of Money, Credit and Banking, v. 29, n. 1, p. 1-16, 1997.

IPEADATA. Expectativa média de Inflação - IPCA - taxa acumulada para os próximos doze meses. Disponível em: 〈http://www.ipeadata.gov.br/Default.aspx > Acesso em: 24 dez. 2019.

IPEADATA. Índice de Confiança do Empresário da Indústria (ICEI): Expectativas. Disponível em: <http://www.ipeadata.gov.br/Default.aspx >. Acesso em: 24 dez. 2019.

JOHNSTON, J. Econometric Methods. New York: McGraw-Hill, 1984.

KATONA, G. Consumer behavior: Theory and findings on expectations and aspirations. The American Economic Review, v. 58, n. 2, p. 19-30, 1968.

KEYNES, J. M. General theory of employment, interest and money. Macmillan Cambridge University Press, 1936.

KHAN, M. S.; SSNHADJI, A. S. Threshold effects in the relationship between inflation and growth. International Monetary Fund, Staff Papers. v. 48, n. 1, p. 1-21, 2001.

LIMA, F.; MARQUES, J. Macroeconomic determinants of unemployment in Brazil: an ARDL Approach. Modern Economy, v. 10, n. 7, p. 1744-1758, 2019.

LOPES, F. Inflação e nível de atividade no Brasil: um estudo econométrico. Pesquisa e Planejamento Econômico, v. 12, n. 3, 1982.

LUDVIGSON, S. Consumer Confidence and Consumer Spending. The Journal of Economic Perspectives, v. 18, n. 2, p. 29-50, 2004.

MALGARINI, M.; MARGANI, P. Psychology, consumer sentiment and household expenditures. Applied Economics, v. 39, n. 13, p. 1719-1729, 2007.

MEDEIROS, G.; PORTUGAL, M.; ARAGÓN, E. Instabilidades na curva de Phillips novo-keynesiana: um estudo empírico para o Brasil. Revista Pesquisa e Planejamento Econômico. v. 47, n. 1, p. 45-76, 2017.

MENDONÇA, M.; SACHSIDA, A.; MEDRANO, L. Inflação versus desemprego: novas evidências para o Brasil. Economia Aplicada, v. 16, n. 3, p. 475-500, 2012. 
NETŠUNAJEV, A.; GLASS, K. Uncertainty and employment dynamics in the euro area and the US. Journal of Macroeconomics, v. 51, p. 48-62, 2017.

PALMA, A.; FERREIRA, D. NAIRU, Inflação e Curva de Phillips no Brasil: novas evidências a partir de um modelo tempo-variante. Estudos Econômicos, v. 47, n. 1, p. 39-63, 2017.

PESARAN, M.; SHIN, Y.; SMITH, R. Bounds testing approaches to the analysis of level relationships. Journal of Applied Econometrics, v. 16, n. 3, p. 289-326, 2001 .

PHELPS, E. Money-wage dynamics and labour market equilibrium. Journal of Political Economy, v. 76, n. 4, p. 678-711, 1968.

POTRICH, A.; VIEIRA, K.; MARION FILHO, P.; FRAGA, L. A confiança do empresariado na indústria de transformação: uma análise em painel. Revista Eletrônica em Gestão, Educação e Tecnologia Ambiental, v. 19, n. 3, p. 98-111, 2015.

PRONI, M. O desemprego na história do pensamento econômico. Revista da $A B E T$, v. 13, n. $1,2014$.

PRONI, M. Teorias do desemprego: um guia de estudo. Texto para discussão $n$. 256. Instituto de Economia da Unicamp, 2015

RENDTEL, U.; SCHARZE, J. Estimates of wage curves for western Germany using a generalised variance-component model. Institute for Employment Research. Nuremberg, v. 29, n. 3, 1996.

SACHSIDA, A. Inflação, desemprego e choques cambiais: uma revisão de literatura sobre a curva de Phillips no Brasil. Revista Brasileira de Economia, v. 67, n. 4, p. 549-559, 2013.

SACHSIDA, A. SCHETTINI, B.; GOUVÊA, R. Inflação, desemprego e choques cambiais: estimativas VAR para a economia brasileira. Análise Econômica, v. 35, n. $67,2017$.

SAREL, M. Nonlinear effects of inflation on economic growth. International Monetary Fund Staff Papers, v. 43, p. 199-215, 1996.

SILVA, J. Macroeconomia: modelo geral de análise macroeconômica. Rio de Janeiro: Publit, 2009.

SUM, V. Unemployment, consumer confidence, business confidence, inflation and monetary policy. SSRN Electronic Journal. September 14, 2012.

TRICHES, D.; FEIJÓ, F. Uma estimação da curva de Phillips híbrida para o Brasil no regime de metas de inflação. Economia Aplicada, v. 21, n. 1, p. 29-43, 2017. 
USMAN, O.; ELSALIH, O. M. Testing the effects of real exchange rate passthrough to unemployment in Brazil. Economies, v. 6, n. 3, p. 49, 2018.

WOOLDRIDGE, J. M. Introdução à econometria: uma abordagem moderna. São Paulo: Thomson Learning, 2007.

ZYLBERSTAJN, H.; NETO, G. B. As teorias do desemprego e políticas públicas de emprego. Estudos Econômicos, v. 29, n. 1, p. 129-149, 1999. 


\section{Apêndice}

Figura 4 - Testes CUSUM e CUSUMSQ de Estabilidade dos Modelos

Modelo 1

Modelo 2
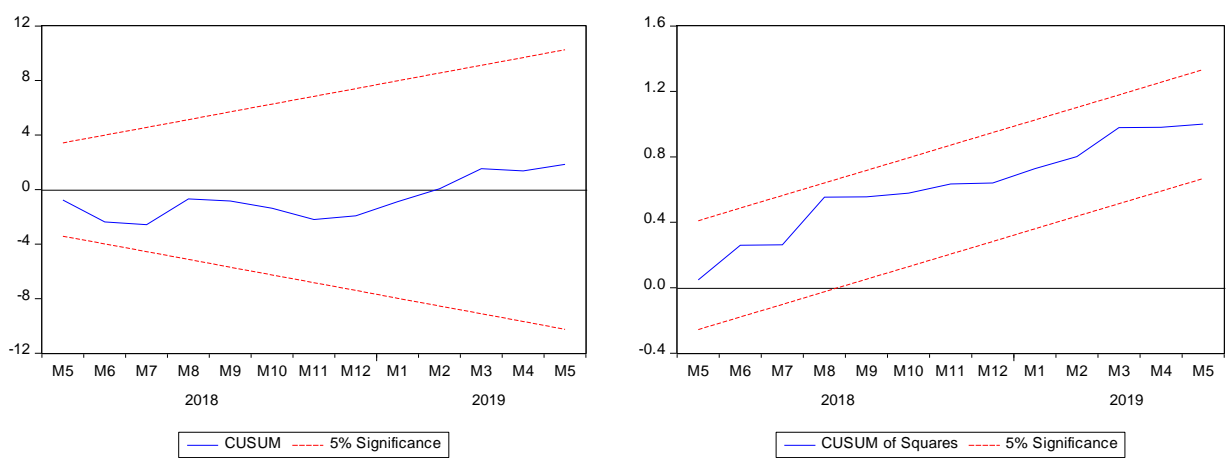

Modelo 3
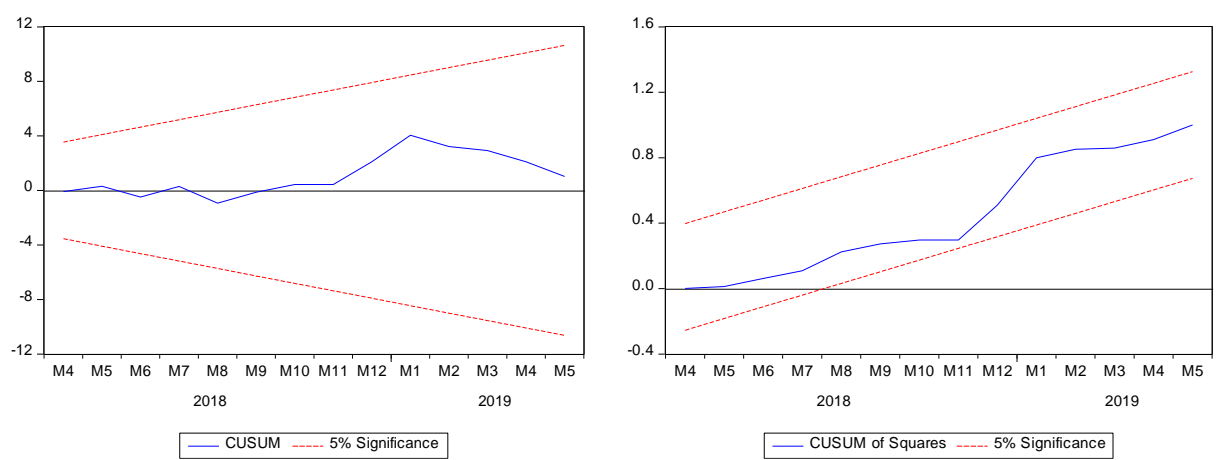

Fonte: Elaboração própria a partir de dados da pesquisa.

Tabela 6 - Variáveis Instrumentais dos Estimadores GMM

\begin{tabular}{ccc}
\hline & Variável dependente: $\operatorname{lnDES}$ & \\
\hline Modelo 1 & Modelo 2 & Modelo 3 \\
\hline $\ln P R O D(-4 \mathrm{a}-6)$ & $\ln P R O D(-2 \mathrm{a}-6)$ & $\ln P R O D(-1 \mathrm{a}-2)$ \\
$\ln I N F(-1 \mathrm{a}-5)$ & $\ln I N F(-1 \mathrm{a}-6)$ & $\ln I N F(-2 \mathrm{a}-6)$ \\
$\ln T A X(-2 \mathrm{a}-6)$ & $\ln T A X(-1 \mathrm{a}-5)$ & $\ln T A X(-1 \mathrm{a}-6)$ \\
- & $\ln I N D(-1 \mathrm{a}-3)$ & $\ln C O M(-2 \mathrm{a}-4)$ \\
\hline
\end{tabular}

Fonte: Elaboração própria a partir dos dados da pesquisa. 
Tabela 7 - Testes de Estacionariedade ADF e PP

\begin{tabular}{|c|c|c|c|c|c|c|c|c|c|}
\hline \multirow[b]{2}{*}{ Séries } & \multicolumn{4}{|c|}{ ADF } & \multicolumn{4}{|c|}{ PP } & \multirow{2}{*}{ Ordem } \\
\hline & Eq & Lag & t-stat & valor crítico & Eq. & Band. & t-stat & valor crítico & \\
\hline $\ln D E S$ & $\mathrm{~N}$ & 11 & -2.9206 & -1.6139 & $\mathrm{~N}$ & 1 & -2.3223 & -1.6142 & $\mathrm{I}(0)$ \\
\hline $\ln P R O D$ & $\mathrm{~N}$ & 0 & -6.9145 & -1.6142 & $\mathrm{~N}$ & 2 & -6.8840 & -1.6142 & $\mathrm{I}(0)$ \\
\hline $\operatorname{lnINF}$ & $\mathrm{N}$ & 0 & -3.6899 & -1.6142 & $\mathrm{~N}$ & 2 & -3.5547 & -2.5849 & $\mathrm{I}(0)$ \\
\hline $\ln T A X$ & $\mathrm{~N}$ & 3 & -4.7285 & -1.6142 & $\mathrm{~N}$ & 6 & -2.8380 & -1.6142 & $\mathrm{I}(0)$ \\
\hline $\operatorname{lnIND}$ & $\mathrm{N}$ & 1 & -2.9531 & -1.6142 & $\mathrm{~N}$ & 3 & -2.7132 & -1.6142 & $\mathrm{I}(0)$ \\
\hline $\ln C O M$ & $\mathrm{~N}$ & 1 & -2.7593 & -1.6142 & $\mathrm{~N}$ & 1 & -2.2449 & -1.6142 & $\mathrm{I}(0)$ \\
\hline
\end{tabular}

Fonte: Elaboração própria.

Notas: "N" (none) significa modelo sem intercepto e tendência. 